\title{
A comprehensive analysis of the cool RCB star DY Persei
}

\author{
L. Začs ${ }^{1}$, S. Mondal ${ }^{2}$, W. P. Chen ${ }^{2}$, A. F. Pugach ${ }^{3}$, F. A. Musaev ${ }^{4,5}$, and O. Alksnis ${ }^{1}$
}

${ }^{1}$ Faculty of Physics and Mathematics, University of Latvia, Rainga bulvāris 19, 1586 Rīga, Latvia e-mail: zacs@latnet.lv

2 Graduate Institute of Astronomy, National Central University, Chung-Li 32054, ROC, Taiwan e-mail: wchen@astro.ncu.edu.tw

3 Main Astronomical Observatory of the National Academy of Sciences of Ukraine, Golosiiv, 252650 Kiev, Ukraine e-mail: pugach@mao.kiev.ua

4 Special Astrophysical Observatory and Isaac Newton Institute of Chile, SAO Branch, 369167 Nizhnij Arkhyz, Russia e-mail: faig@sao.ru

5 ICAMER, National Academy of Sciences of Ukraine, 361605 Peak Terskol, Kabardino-Balkaria, Russia

Received 12 December 2006 / Accepted 4 June 2007

\section{ABSTRACT}

\begin{abstract}
Context. Theoretically, the number of cool Galactic R Coronae Borealis stars (RCBs) should be greater than the warm RCBs, however to date, only a few candidates have been detected.

Aims. Observations of the extremely cool RCB candidate, DY Per, and the anonymous nearby star (the "Taipei star") are presented to specify its fundamental parameters and evolutionary status.

Methods. CCD BVRI photometry and low-resolution spectroscopy at deep light decline was carried out in 2004. A high-resolution spectrum was gathered near the maximum light in 2002, and a qualitative analysis was made relative to the typical carbon stars of various types.

Results. Near the light maximums, the nearby anonymous star with a separation of $\sim 2$ ". 5 reported by Začs et al. (2005a, A\&A, 438, L13) was outshined by DY Per. However, in the $B$ and $V$ band the components are resolved at deep light decline and the nearby star is somewhat brighter in $B$ than DY Per itself. $B V(R)$ individual magnitudes of the components were estimated using PSF photometry for the first time. The light decline in 2004 was the deepest ever observed for DY Per, $B=18.16$ mag. The individual colour indices of DY Per at light decline, $(B-V)=2.35$ and $(V-R) \simeq 1.9$, are not typical for carbon stars. In the spectrum, absorption features of $C_{2}$ and $\mathrm{CN}$ molecules prevail, however, prominent $C_{2}$ absorption bands are significantly veiled at deep-light decline and a broad $\left(F W H M \sim 600 \mathrm{~km} \mathrm{~s}^{-1}\right)$ emission feature of NaID12 is visible. Emissions are suspected in the $C_{2}(1,0)$ bandhead and in Ca I line at $4227 \AA$ A. Analysis of DY Per relative to the sample of carbon stars, verifies the significant hydrogen deficiency, high carbon abundance, relatively high ${ }^{12} \mathrm{C} /{ }^{13} \mathrm{C}$ ratio and solar metallicity. Thus DY Per could be a prototype of cool extreme Galactic RCBs. The nearby star does not vary appreciably in the $B$ and $V$ bands, $B=17.8 \mathrm{mag}$. With the observed colour indices $(B-V)=1.00$ and $(V-R) \simeq 0.6$, it may be a G0 dwarf not physically related to DY Per, although the distances to both stars are similar, $d \sim 1.5 \mathrm{kpc}$.
\end{abstract}

Key words. stars: carbon - stars: winds, outflows - stars: fundamental parameters - stars: circumstellar matter stars: individual: DY Per - stars: evolution

\section{Introduction}

$\mathrm{R}$ Coronae Borealis (RCB) stars are a rare type of hydrogendeficient, carbon-rich, evolved stars which undergo strong, irregular brightness variations in the optical region (see Clayton 1996, for a detailed review). Only about 50 RCBs have been identified in the Milky Way. Theoretically, two major scenarios have been proposed to account for the main characteristics of RCBs, one involving the merger of two white dwarfs, and the other involving a final helium shell flash in an evolved planetary nebula central star (Iben et al. 1996). Both of the evolutionary scenarios predict significantly more Galactic RCBs than are detected. Iben et al. (1996) estimated that the final flashes should produce from 30 to 2000 Galactic RCB stars at any given time. In the case of the binary merger scenario, the number of RCBs should be about 300 . The evolutionary models imply relative populations of RCBs with $T_{\text {eff }}=5000,6000$, and $7000 \mathrm{~K}$ of 30:5:1 (Lawson et al. 1990, and references therein), however, most of the known Galactic RCBs fall into the warmest subgroup. The apparent lack of cool RCB stars is not clear, however, this could be a selection effect.

DY Persei (DY Per) was suspected to be a cool RCB star by photometric study (Alksnis et al. 2002, and references therein). A number of sudden light declines typical for RCB variables were detected for this cool carbon (CGCS 372; C 5,4) star along with semiregular large amplitude pulsations with a period of 792 days. Its temperature is much lower than the average of other (warm) RCB stars. Keenan \& Barnbaum (1997; hereafter KB97) estimated $T_{\text {eff }} \simeq 3500 \mathrm{~K}$ using the standard criteria of spectral classification and suspected a giant luminosity for DY Per. The high-resolution spectroscopy revealed strong carbon features and a relatively high ${ }^{12} \mathrm{C} /{ }^{13} \mathrm{C}$ ratio, whereas neutron-capture elements are not significantly enhanced in the atmosphere of DY Per (Dominy 1985; KB97; Začs et al. 2005a). Začs et al. (2005a) detected an anonymous star about $2 ., 5$ from DY Per and confirmed the existence of ejected clouds. Here we present a comprehensive analysis of the original data of DY Per acquired in recent years with a special emphasis on the observations near the deep light decline in late 2004. 
Alcock et al. (2001) found four stars similar to DY Per among the eight RCBs discovered in the Large Magellanic Cloud (LMC). For the RCBs in the LMC they found a range of absolute magnitudes $M_{V}$ from -2.5 to $-5 \mathrm{mag}$ and a temperature- $M_{V}$ relationship showing the warm RCBs to be brighter than the cool $\left(T_{\text {eff }} \sim 5000 \mathrm{~K}\right)$ ones. However, the DY Per-like stars of LMC show evidence for significant enhancement of ${ }^{13} \mathrm{C}$ and a small amplitude of photometric pulsations (Alcock et al. 2001). Thus the evolucionary status of DY Per and DY Per-like stars of LMC could be different. Kramer et al. (2005) detected DY Per-like star (MSX SMC 155) in the Small Magellanic Cloud (SMC). The studies of long-term photometric variations of 18 carbon stars in Cygnus, revealed another star, V1983 Cyg, to be a cool Galactic RCBs candidate (Alksnis 2003). Zaniewski et al. (2005) identified four relatively cool $\left(T_{\text {eff }}<6000 K\right)$ RCBs in the Galactic bulge using the MACHO project photometry database. Additional observations and a comprehensive analysis are needed to understand the relationship and evolutionary status of these objects. The mass ejection, fundamental parameters and detailed chemistry should be confirmed using high resolution spectroscopy and atmospheric models.

Alcock et al. (2001) estimated the number of Galactic RCBs to be about 3200 based on the number of observed RCB stars in the LMC and extropolating this to the Galaxy. Zaniewski et al. (2005) recently acknowledged the predominance of cool RCB stars in the Galaxy and the selection bias for previously discovered RCBs. Unfortunately, these conclusions were based on very small sample of RCBs candidate stars discovered in the MACHO fields: 13 RCB candidate stars in the LMC and four cool RCB candidate stars in the Galactic bulge with effective temperatures lower than $6000 \mathrm{~K}$.

\section{Observations and data reduction}

The imaging photometry of DY Per was taken with the Lulin 1-m telescope (LOT), equipped with a Princeton Instruments PI 1300B CCD camera. The $\mathrm{f} / 8$ optics of the telescope provides a pixel scale of 0.516 , and an FOV about $11^{\prime}$ on the focal plane. DY Per was sequentially monitored in the BVRI bands from early October 2004 through late January 2005. Typical seeing was about $2^{\prime \prime}$. Exposure times ranged from $20 \mathrm{~s}$ in the $I$ and $R$ bands, $90 \mathrm{~s}$ in the $V$ band, to $300 \mathrm{~s}$ in the $B$ band. The Landolt standards, SA92 \#245, 248, 249 and 250, were used for flux calibration. The images were processed following standard procedures of bias and dark subtraction and flat fielding using the IRAF package. PSF photometry was used to estimate individual fluxes of DY Per and the close anonymous star in the $B$ and $V$ bands. Aperture photometry was used to estimate the combined flux of the pair in the $R$ and $I$ bands, except for 3 nights of relatively good sky conditions, it was possible to separate the individual components in the $R$ band. To aid future observations, the photometry for several possible reference stars (Fig. 1) near DY Per was also carried out and is reported here.

Low-resolution spectra for DY Per were observed with the Nasmyth spectrometer SPEM on the 2.6-m Shain telescope at the Crimean Astrophysical Observatory. The spectra were obtained near the deep light decline in the spectral region from about 4200 to $7200 \AA$ with a resolution of about $\sim 2.2 \AA$. The standard stars HR 153 and 30 Per were observed to provide flux calibration. The spectral region observed in one exposure was about $1100 \AA$. The log of observations is provided in Table 1 .

High-resolution spectra for DY Per and most of the comparison carbon stars were obtained with the Coudé échelle

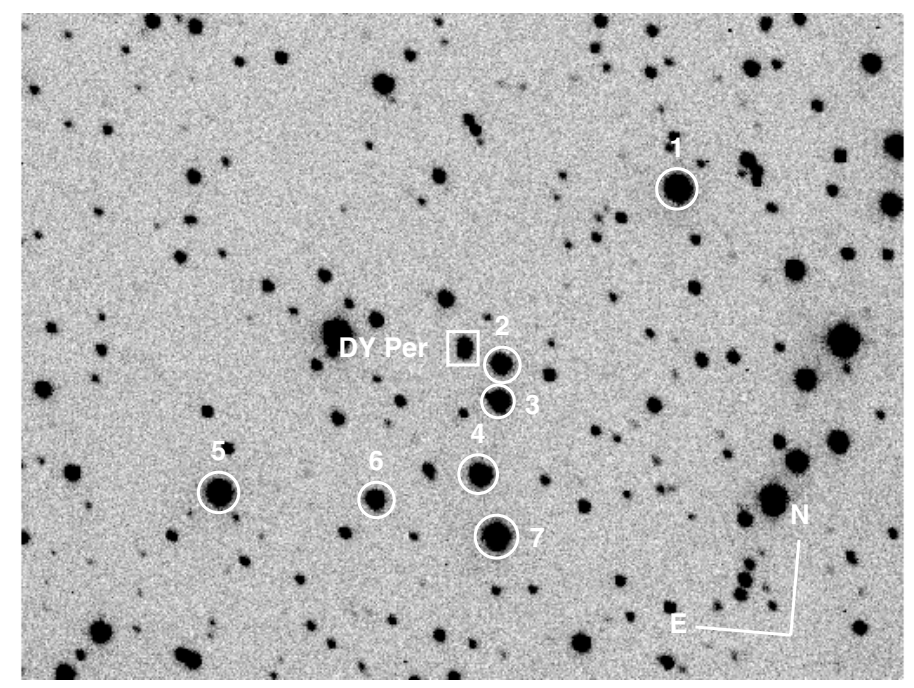

Fig. 1. The $5^{\prime} \times 5^{\prime}$ field around DY Per (square box) in the $R$ band. Reference stars are encircled and labeled.

Table 1. The log of low-resolution spectroscopic observations. Dates of observations, central wavelengths, exposures, and maximal $S / N$ ratios are given.

\begin{tabular}{ccccc}
\hline \hline Data & Star & $\lambda_{c}(\AA)$ & Exposure $(\min )$ & $S / N$ \\
\hline 26.09 .2003 & DY Per & 4400 & 20 & 136 \\
& DY Per & 5500 & 20 & 264 \\
& DY Per & 6560 & 20 & 280 \\
& HR 153 & 5500 & 1 & 404 \\
19.09 .2004 & DY Per & 5500 & $2 \times 30$ & 35 \\
& 30 Per & 5500 & $3 \times 0.5$ & 407 \\
20.09 .2004 & DY Per & 4400 & 30 & 21 \\
& DY Per & 5500 & 30 & 34 \\
21.09 .2004 & 30 Per & 5500 & $3 \times 0.5$ & 366 \\
& DY Per & 6560 & 30 & 49 \\
& DY Per & 7250 & 20 & 37 \\
\hline
\end{tabular}

spectrometer MAESTRO on the $2 \mathrm{~m}$ telescope at the Observatory on the Terskol Peak in Northern Caucasus equipped with a CCD detector and a resolving power of $\sim 45000$. A total exposure time of $7200 \mathrm{~s}$ was made for DY Per on 18 November 2002. The spectrum covered from 3600 to $10200 \AA$ in 85 wavelength bands overlapping shortward of $\mathrm{H}_{\alpha}$. Each region spanned from 50 to $140 \AA$. Because of the energy distribution in carbon stars, the signal-to-noise $(S / N)$ ratio in the spectrum of DY Per shortward of $4800 \AA$ is very poor. The reduction of spectra was performed with the standard DECH20T routines. The moments of spectroscopic observations relative to the light declines are indicated in Fig. 2.

\section{Analysis}

\subsection{Photometry}

The BVRI magnitudes of seven reference stars were derived in the field around DY Per and are reported, together with their uncertainties $(\sigma)$ and the number of observations $(N)$, in Table 2 . The uncertainties are calculated from the errors of individual measurement plus the standard deviation in the number of observations. An identification chart for the reference stars is shown in Fig. 1. All these stars are relatively bright, and should serve as 


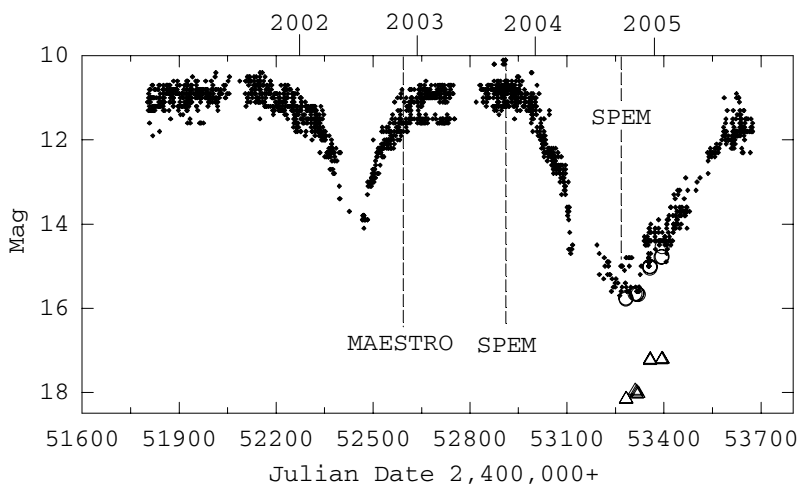

Fig. 2. Recent light variations of DY Per according to the AAVSO data (dots) in 2000 days. Measurements in $B$ (triangles) and $V$ (open circles) bands observed with the LOT are shown. The moments of highresolution (MAESTRO) and low-resolution (SPEM) spectroscopy are indicated by the dashed lines.

useful comparison objects for photometric monitoring of DY Per in the future.

The detection of a nearby anonymous star (the "Taipei star") some 2'.5 from DY Per and preliminary magnitudes in $B$ and $V$ bands for one night at deep light decline in 2004 have been reported previously (Začs et al. 2005a). Photometric monitoring for both DY Per and the anonymous star continued for approximately 4 months. Here the individual magnitudes in $B$ and $V$ bands, in which the pair are clearly resolved, are presented. At longer wavelength $R$ and $I$ bands, however, DY Per dominated the fluxes so the pair could no longer be resolved. In such cases the combined magnitudes of the pair are given. In 3 nights under good seeing conditions the 2 stars were resolved in $R$. These photometric results are summarized in Tables 3 and 4. In Table 3 the combined magnitudes of the pair are also provided in $R$ and $I$ bands using IRAF/APERTURE photometry. DY Per varied from $B=18.2-17.2 \mathrm{mag}\left(\Delta m_{B} \sim 0.95\right), V=15.8-$ $14.5 \mathrm{mag}\left(\Delta m_{V} \sim 1.2\right), R=13.95-12.7 \mathrm{mag}\left(\Delta m_{R} \sim 1.2\right)$, and $I=11.9-10.9 \mathrm{mag}\left(\Delta m_{I} \sim 1.0\right)$. The light decline in 2004 was the deepest ever recorded for DY Per. As seen in Table 4 the nearby star does not vary appreciably in $B$ or $V$, so the combined flux changes in $R$ and $I$ bands must be due mainly to DY Per. With no prior knowledge of the existence of the anonymous star, previous photometric measurements of DY Per, in particular toward short wavelengths, must have been influenced, so should be interpreted with care.

The anonymous star, judging from its observed colour indices $(B-V)=1.0$ and $(V-R) \simeq 0.6$ (Table 4$)$, is probably not physically related to DY Per. The star is seen toward the open cluster Trumpler 2, which has an interstellar extinction $E(B-V)=0.32 \mathrm{mag}$ (Kharchenko et al. 2005). Thus the intrinsic colour for the anonymous star could be $(B-V)_{0} \simeq 0.7$ which is close to that for a G5 main sequence star of solar chemical composition (Johnson 1966). The distance of such a dwarf with $M_{V}=4.8$ should be about $1.6 \mathrm{kpc}$ for the adopted total interstellar reddening of $A_{V} \simeq 1.0 \mathrm{mag}$ (using $A_{V} \simeq 3.1 E(B-V)$ ). Thus the anonymous star is much farther than Trumpler 2 which is located at a distance of 649 pc (Kharchenko et al. 2005). The reddening in the field around DY Per provided by Neckel \& Klare (1980) at such a distance should be $A_{V} \simeq 1.4$ mag. We adopted as an iterated solution $A_{V} \simeq 1.3 \mathrm{mag}$ which leads to the distance of about $1.4 \mathrm{kpc}$.

\subsection{Low-resolution spectroscopy}

\subsubsection{Absorption features}

The majority of RCBs spectroscopically resemble the F-G Ib supergiants with $T_{\text {eff }}=7000 \pm 1000 \mathrm{~K}$, but with weaker Balmer lines and $\mathrm{CH}$ band than in normal supergiants due to the hydrogen deficiency (see Clayton 1996). C I lines and $C_{2}$ molecular bands are stronger due to the carbon excess. The absorption lines are usually weakened at a light decline. An extreme case was observed for V854 Cen, in which the continuum was featureless by absorption lines at the light decline.

The low-resolution spectra of DY Per observed near light maximum and at deep light decline were compared to inspect the typical spectroscopic peculiarities of RCBs. The absorption spectrum of DY Per, owing to the low photospheric temperature and the carbon enhancement, is very crowded with molecular lines of carbon-bearing molecules. Certainly determination of the continuum becomes a serious problem and any quantitative analysis would be plagued. In fact the continuum is not detectable in the medium resolution spectra of such stars due to the enormous number of blending lines. Therefore a pseudocontinuum was defined using the wavelength regions which are relatively free from strong absorptions. An inspection of the lowresolution spectra for DY Per relative to the synthesized spectra shows that prominent absorption features of $C_{2}$ Swan and $\mathrm{CN}$ Red systems prevail through the observed spectral region (Fig. 3).

As can be seen in Fig. 3, the $C_{2}$ absorption bands in the spectrum of DY Per are significantly veiled at the deep light decline. This is in agreement with what has been observed for warm RCBs stars. However, in the case of DY Per the spectrum was influenced by the nearby anonymous star because both stars were not resolved during the spectroscopic observations with moderate seeing conditions. We did a crude simulation of the combined spectrum in the blue spectral region at deep light decline when the influence of the nearby star was the largest. According to the photometric observations both stars in the $B$ band $\left(\lambda_{\text {eff }}=4360 \AA, \Delta \lambda=940 \AA\right.$ ) are of approximately similar brightness, $B=18.16 \mathrm{mag}$, and $B=17.75 \mathrm{mag}$, in October 2004 when the spectra were observed. Thus the observed flux at deep light decline in the blue spectral region should be the sum of two components with a similar weight. In the visible and (especially) in the red the flux of DY Per is less affected. Near light maxima, the influence of the anonymous star on DY Per should be negligible. The combined relative flux (DY Per + anonymous star; Fig. 4) at deep light decline in the blue spectral region was gathered by adding the normalized spectrum of DY Per near light maximum and a synthetic spectrum $(\mathrm{G} 5 \mathrm{~V})$ appropriate for the anonymous star. It is evident that the shape of the calculated combined spectrum agrees in general with that observed. Thus our simple model explained the veiling of strong absorption features in the spectrum of DY Per at deep light decline.

\subsubsection{Synthetic spectra}

Synthetic spectra of the anonymous star and the comparison stars in selected wavelength regions were calculated using the standard code STARSP developed by $V$. Tsymbal, convolving with the instrumental profile. The solar atmospheric model was adopted for the anonymous star with suitable parameters of $T_{\text {eff }}=5660 \mathrm{~K}, \log g=4.44(\mathrm{cgs})$, and $\xi_{\mathrm{t}}=1.2 \mathrm{~km} \mathrm{~s}^{-1}$. The Kurucz (1993) models were used for comparison stars with the effective temperature above $3500 \mathrm{~K}$. Two atmospheric models of 
Table 2. BVRI magnitudes of reference stars in the field around DY Per. The uncertainties $(\sigma)$ and the number of observations $(N)$ are given for each band.

\begin{tabular}{ccccccccc}
\hline \hline Star & $B(\sigma)$ & $N_{B}$ & $V(\sigma)$ & $N_{V}$ & $R(\sigma)$ & $N_{R}$ & $I(\sigma)$ & $N_{I}$ \\
\hline 1 & $14.395(0.015)$ & 12 & $13.474(0.008)$ & 12 & $12.960(0.005)$ & 16 & $12.533(0.006)$ & 12 \\
2 & $15.848(0.041)$ & 12 & $14.009(0.011)$ & 12 & $12.814(0.009)$ & 16 & $11.498(0.012)$ & 12 \\
3 & $15.646(0.028)$ & 12 & $15.017(0.012)$ & 12 & $14.717(0.013)$ & 16 & $14.359(0.026)$ & 12 \\
4 & $15.351(0.019)$ & 12 & $14.480(0.011)$ & 12 & $14.009(0.007)$ & 16 & $13.579(0.015)$ & 12 \\
5 & $14.693(0.026)$ & 12 & $14.191(0.007)$ & 12 & $13.978(0.006)$ & 16 & $13.779(0.010)$ & 12 \\
6 & $16.148(0.020)$ & 12 & $14.776(0.012)$ & 12 & $13.970(0.007)$ & 16 & $13.183(0.011)$ & 12 \\
7 & $14.490(0.007)$ & 12 & $13.503(0.005)$ & 12 & $12.961(0.004)$ & 16 & $12.488(0.009)$ & 12 \\
\hline
\end{tabular}

Table 3. $B V R I$ photometry of DY Per. The color indexes $(B-V),(V-R)$ and standard deviations are also given.

\begin{tabular}{|c|c|c|c|c|c|c|c|}
\hline Date & $\begin{array}{c}\text { JD } \\
(2453+)\end{array}$ & $\begin{array}{c}B \\
\text { (individual) }\end{array}$ & $\begin{array}{c}V \\
\text { (individual) }\end{array}$ & $\begin{array}{c}R \\
\text { (combined) }\end{array}$ & $\begin{array}{c}I \\
\text { (combined) }\end{array}$ & $\overline{B-V}$ & $\overline{V-R}$ \\
\hline $04-10-2004$ & 283 & $18.16 \pm 0.023$ & $\begin{array}{l}15.76 \pm 0.011 \\
15.79 \pm 0.010\end{array}$ & $\begin{array}{l}13.86 \pm 0.002 \\
13.85 \pm 0.003 \\
13.86 \pm 0.003\end{array}$ & $\begin{array}{l}11.91 \pm 0.001 \\
11.93 \pm 0.003 \\
11.93 \pm 0.002\end{array}$ & $2.38 \pm 0.027$ & \\
\hline 01-11-2004 & 311 & $17.96 \pm 0.030$ & $15.66 \pm 0.017$ & $\begin{array}{c}13.58 \pm 0.003 \\
13.67 \pm 0.004(\mathrm{psf})\end{array}$ & $11.65 \pm 0.001$ & $2.30 \pm 0.039$ & $1.99 \pm 0.025$ \\
\hline 06-11-2004 & 316 & $17.99 \pm 0.015$ & $\begin{array}{l}15.66 \pm 0.019 \\
15.67 \pm 0.018\end{array}$ & $\begin{array}{c}13.58 \pm 0.003 \\
13.64 \pm 0.003 \\
13.77 \pm 0.011(\mathrm{psf})\end{array}$ & $\begin{array}{c}1.64 \pm 0.001 \\
11.68 \pm 0.002\end{array}$ & $2.36 \pm 0.034$ & $1.90 \pm 0.032$ \\
\hline & & $18.05 \pm 0.013$ & $15.65 \pm 0.022$ & $\begin{array}{c}13.63 \pm 0.003 \\
13.76 \pm 0.009(\mathrm{psf})\end{array}$ & $11.69 \pm 0.002$ & & \\
\hline 11-11-2004 & 321 & $18.03 \pm 0.009$ & $15.68 \pm 0.007$ & $13.67 \pm 0.002$ & & $2.35 \pm 0.011$ & \\
\hline $17-12-2004$ & 357 & $17.22 \pm 0.012$ & $15.02 \pm 0.011$ & $\begin{array}{c}13.10 \pm 0.003 \\
13.21 \pm 0.018(\mathrm{psf})\end{array}$ & $11.18 \pm 0.002$ & $2.20 \pm 0.040$ & $1.82 \pm 0.030$ \\
\hline & $\begin{array}{l}357 \\
357\end{array}$ & $\begin{array}{l}17.22 \pm 0.017 \\
17.25 \pm 0.016\end{array}$ & $\begin{array}{l}15.05 \pm 0.020 \\
15.01 \pm 0.020\end{array}$ & & $\begin{array}{l}11.17 \pm 0.002 \\
11.17 \pm 0.002\end{array}$ & & \\
\hline 21-01-2005 & 392 & $17.23 \pm 0.017$ & $14.79 \pm 0.005$ & $12.80 \pm 0.001$ & $11.01 \pm 0.001$ & $2.45 \pm 0.017$ & \\
\hline $\begin{array}{l}24-01-2005 \\
28-01-2005\end{array}$ & 395 & $17.21 \pm 0.017$ & $\begin{array}{l}14.78 \pm 0.018 \\
14.54 \pm 0.006\end{array}$ & $\begin{array}{l}12.73 \pm 0.002 \\
12.65 \pm 0.001\end{array}$ & $10.90 \pm 0.001$ & $2.43 \pm 0.024$ & \\
\hline
\end{tabular}

Table 4. $B V R$ psf photometry of the anonymous star.

\begin{tabular}{ccccccc}
\hline \hline Date & $\begin{array}{c}\text { JD } \\
(2453+)\end{array}$ & $B$ & $V$ & $R$ & $B-V$ & $V-R$ \\
& 283 & $17.75 \pm 0.017$ & $16.78 \pm 0.021$ & & $0.96 \pm 0.033$ & \\
$04-10-2004$ & & & $16.80 \pm 0.019$ & & & \\
$01-11-2004$ & 311 & $17.87 \pm 0.029$ & $16.85 \pm 0.028$ & $16.17 \pm 0.06$ & $1.02 \pm 0.053$ & $0.68 \pm 0.07$ \\
& & & $16.86 \pm 0.034$ & & & \\
$06-11-2004$ & 316 & $17.81 \pm 0.012$ & $16.86 \pm 0.022$ & $16.23 \pm 0.018$ & $1.01 \pm 0.037$ & $0.63 \pm 0.04$ \\
& 316 & $17.90 \pm 0.011$ & $16.84 \pm 0.025$ & $16.21 \pm 0.016$ & & \\
$11-11-2004$ & 321 & $17.84 \pm 0.010$ & $16.81 \pm 0.025$ & & $1.03 \pm 0.027$ & \\
$17-12-2004$ & 357 & $17.82 \pm 0.017$ & $16.84 \pm 0.025$ & $16.26 \pm 0.025$ & $1.00 \pm 0.058$ & $0.57 \pm 0.04$ \\
& 357 & $17.82 \pm 0.023$ & $16.85 \pm 0.024$ & & & \\
$21-01-2005$ & 357 & $17.87 \pm 0.023$ & $16.79 \pm 0.029$ & & & \\
$24-01-2005$ & 395 & $17.84 \pm 0.030$ & $16.82 \pm 0.028$ & & $1.02 \pm 0.041$ & \\
$28-01-2005$ & & $17.81 \pm 0.028$ & $16.85 \pm 0.029$ & & $0.97 \pm 0.040$ & \\
\hline
\end{tabular}

carbon stars (warm and cool) calculated using SCMARCS code were adopted for the atmospheric parameters and $\mathrm{C} / \mathrm{O}$ ratio $\left(T_{\mathrm{eff}}\right.$, $\log g, \mathrm{C} / \mathrm{O})$ of $(3700 \mathrm{~K}, 0.4,1.07)$ and $(2800 \mathrm{~K},-0.6,1.04)$, respectively. The synthetic spectra of $C_{2}$ and $\mathrm{CN}$ molecules were calculated by $\mathrm{M}$. Schmidt in all observed spectral region for these two atmospheric models using spectrum synthesis technique (see Začs et al. 2005b).

\subsubsection{Emission lines}

A rich narrow-line $\left(\sim 50 \mathrm{~km} \mathrm{~s}^{-1}\right)$ emission spectrum appears in RCBs when the photospheric light is extinguished by the forming dust cloud (see Clayton 1996, and references therein). The emission spectrum in the visible spectral region consists of many lines of neutral and singly ionized metals which are slightly blueshifted $\left(\sim 10 \mathrm{~km} \mathrm{~s}^{-1}\right)$ from the stellar radial velocity. Some of these emission lines (e.g. low excitation multiplets of Sc II and Ti II) remain strong for an extended period of time. The late-decline broad-line $\left(100-200 \mathrm{~km} \mathrm{~s}^{-1}\right)$ spectrum is dominated by five strong lines, $\mathrm{Ca}$ II $\mathrm{H}$ and $\mathrm{K}$, the $\mathrm{Na}$ I D lines and $\mathrm{He} \mathrm{I}$ line at $3888 \AA$. This broad-line emission spectrum remains visible until the star returns to maximum light. In some declines, the Swan bands of $C_{2}$ are seen in emission. Rao \& Lambert (1993) discussed the spectrum of V854 Cen with sharp chromospheric 


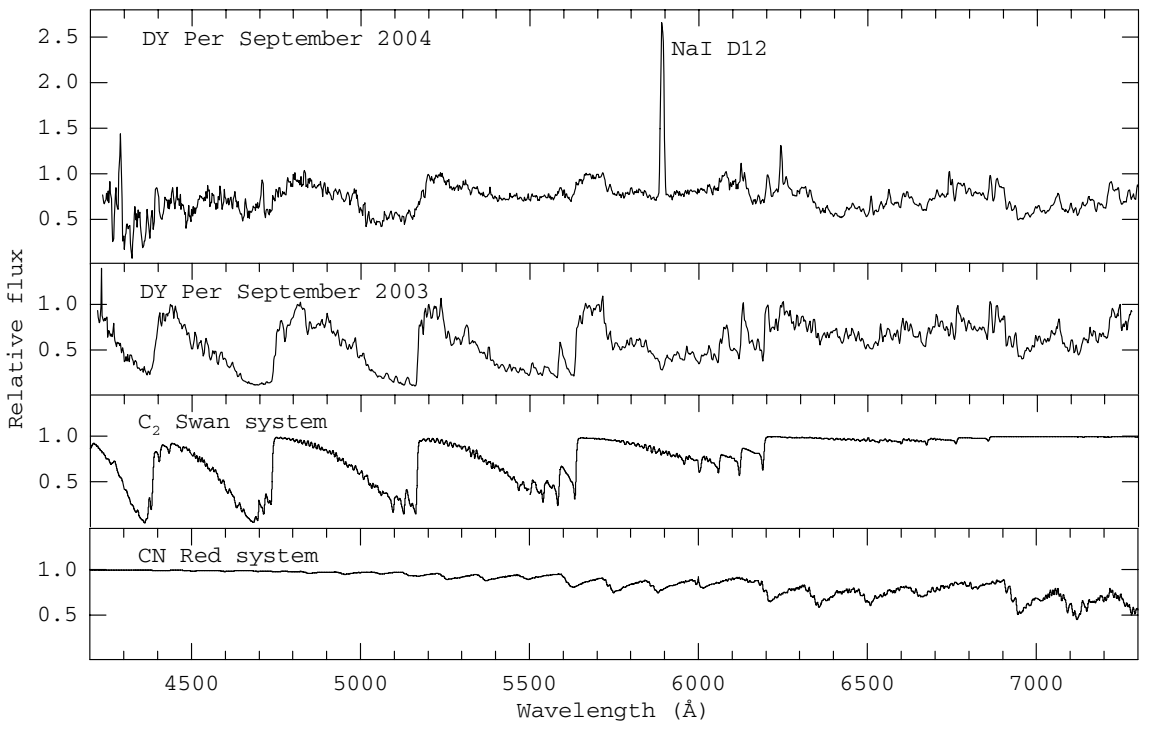

Fig. 3. Low-resolution spectra of DY Per in the spectral region from 4200 to $7300 \AA$ A observed near the light maximum in 2003 and at deep light decline in 2004. The spectra are normalized to pseudo-continuum. Calculated spectra of $C_{2}$ Swan system and CN Red system for typical warm $(3700 \mathrm{~K})$ carbon stars are given for comparison purposes.

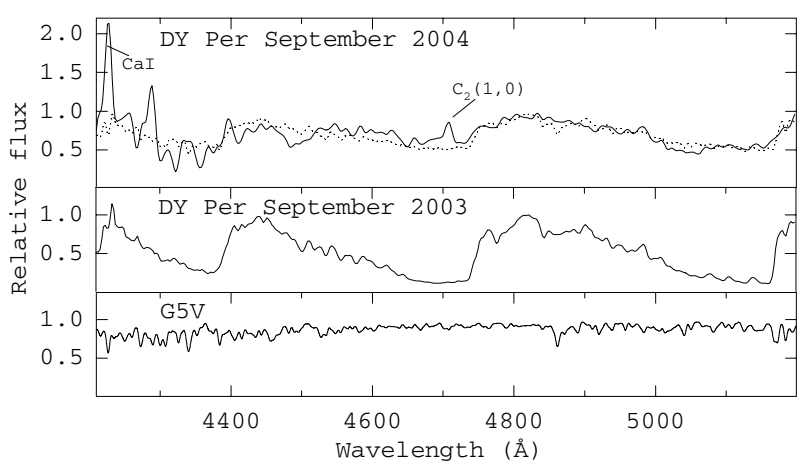

Fig. 4. Low-resolution spectrum of DY Per observed at deep light decline (upper panel; solid line) in the spectral region from 4200 to $5200 \AA$ A. Dotted line represents the sum of two spectra: the observed spectrum of DY Per near light maximum (middle panel) and the synthesized spectrum of $\mathrm{G}$ dwarf star of solar chemical composition (lower panel).

lines and broad emission lines $\left(F W H M=200-300 \mathrm{~km} \mathrm{~s}^{-1}\right)$ of Ca II $\mathrm{H}$ and $\mathrm{K}, \mathrm{Na}$ I D, $\mathrm{H}_{\alpha}$, and the $C_{2}$ Swan bands as well as the forbidden lines of [NII], [OI], and [SII]. Very few quantitative measurements have been made of the emission line strengths during declines.

The spectra of DY Per were inspected and a few emission lines were found. The most prominent feature at deep light decline is a broad $\left(F W H M \sim 600 \mathrm{~km} \mathrm{~s}^{-1}\right)$ emission feature of Na I D12 (Fig. 3). A Ca I line at $4227 \AA$ is suspected to be present in emission (Fig. 4). The simulations of the combined (DY Per + nearby star) spectrum in the blue spectral region revealed possible emission in the $C_{2}(1,0)$ bandhead (Fig. 4). The presence of another emission feature is disputable because of the problems in removal of cosmic rays in the data.

\subsubsection{Absolute flux}

The standard stars with known absolute flux (HR 153, 30 Per) were observed under best atmospheric conditions to estimate the energy distribution of DY Per. The absolute energy distribution

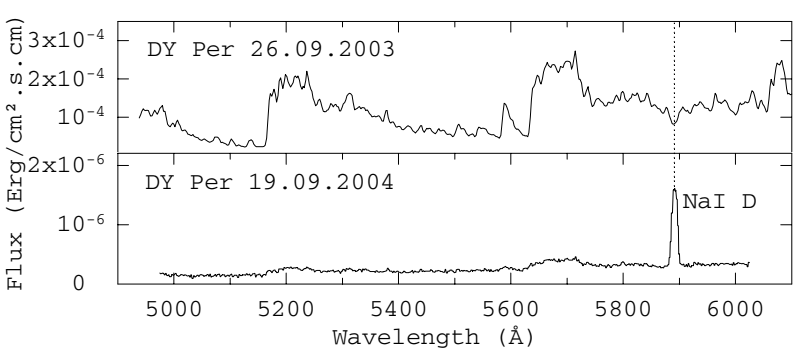

Fig. 5. Absolute energy distribution in the spectrum of DY Per around $5500 \AA$ A near light maximum and at deep light decline.

for DY Per in the visual spectral region around $5500 \AA$ is shown in Fig. 5. Averaged values of the extinction coefficients calculated by Metlov (2004) were adopted. A monochromatic magnitude at $5500 \AA$ was calculated using absolute flux near the light maximum and at deep decline, $m_{5500}=9.10$ and $15.42 \mathrm{mag}$, respectively. However, the absolute flux and monochromatic magnitudes are affected by the nearby anonymous star, especially at light decline.

\subsection{High-resolution spectroscopy}

The use of traditional methods of atmospheric models to estimate the fundamental parameters and abundances for DY Per has been plagued due to the lack of reliable atmospheric models. The crowded spectrum of this cool carbon-rich star significantly enhanced the problems of fine spectroscopy. Therefore a comprehensive analysis presented here based mainly on the comparison of high-resolution spectra of similar resolution for DY Per and carbon stars of various types: U Hya, UV Cam, W CMa, $\mathrm{R}$ Lep, and $\mathrm{BD}+57^{\circ} 2161$. Basic data of these comparison stars are summarized in Table 5. The data are collected from various sources: temperature and luminosity from Bergeat et al. (2002) and KB97, abundances from Abia et al. (2002), Lambert et al. (1986), KB97, and Začs et al. (2005b). Although the atmosphere of DY Per is warmer than that for typical cool N-type carbon stars, the spectrum is more complicated than those for U Hya and W CMa. 
Table 5. Basic data for DY Per and the comparison stars collected from literature.

\begin{tabular}{ccccccccc}
\hline \hline Star & CGCS & Var, P & Sp.type & $T_{\text {eff }}$ & $M_{\text {bol }}$ & {$[\mathrm{Fe}]$} & {$[\mathrm{s} / \mathrm{Fe}]$} & ${ }^{12} \mathrm{C} /{ }^{13} \mathrm{C}$ \\
\hline DY Per & 372 & SRb, 792 d & R8, C 4,5 & $\sim 3500$ & giant? & solar & solar & contradictory \\
UV Cam & 608 & SRb & R8, C 5,3J & 3495 & -4.85 & +0.2 & +0.1 & 4 \\
R Lep & 833 & M, 427 d & N6e, C 7,6e & 2290 & -4.87 & +0.2 & - & 62 \\
W CMa & 1565 & Lb & N, C 6,3 & 2960 & -5.42 & +0.3 & +0.6 & 53 \\
U Hya & 2803 & SRb, 450 d & N2, C 7,3 & 2965 & -3.93 & -0.1 & +1.0 & 32 \\
BD +57 2161 & 4784 & var? & R0, C 3,1 & 4555 & -1.53 & -0.2 & +1.5 & $\sim 10$ \\
\hline
\end{tabular}

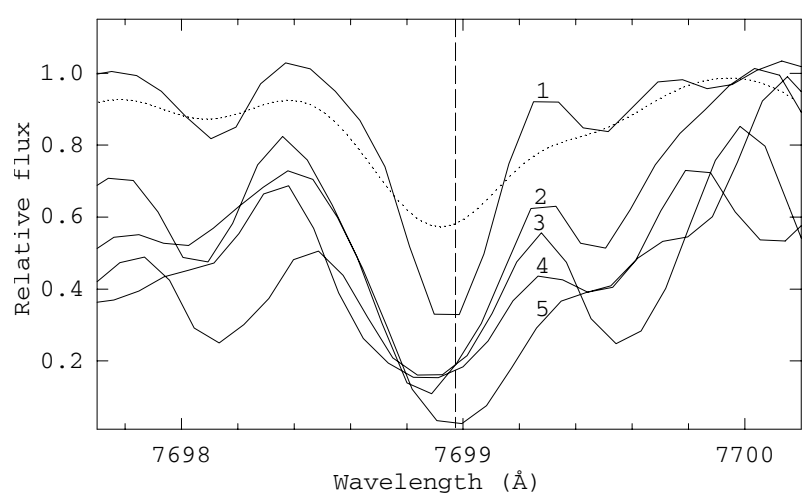

Fig. 6. High-resolution spectrum of DY Per (3) in the spectral region around K I line at $7698.98 \AA$ marked by dashed line. The spectra of carbon stars with different effective temperature, $\mathrm{BD}+57^{\circ} 2161$ (1), U Hya (2), UV Cam (4), and R Lep (5) are provided for comparison purposes. The dotted line represents synthetic spectrum of CN Red system calculated for typical warm $(3700 \mathrm{~K})$ carbon star.

During the spectroscopic observations near maximum light the nearby stars, DY Per and the anonymous star, were not resolved. However, in the red spectral region near maximum light influence of the anonymous star to the spectrum of DY Per should be negligible - DY Per is much cooler and brighter. All spectra provided in this paper have been shifted in wavelength to correct for the stellar radial velocities.

\subsubsection{Atmospheric parameters and luminosity}

Determination of the temperature and surface gravity for cool carbon star is nontrivial. The atomic and molecular lines formed in stellar atmospheres are potential termometers. The spectroscopic methods available for estimating surface gravities of $\mathrm{G}$ and $\mathrm{K}$ giants based on the study of ionization equilibria, dissociation equilibria, and damping wings of strong lines. However, the heavily blanketed spectra of carbon stars camouflage weak lines of iron group ions useful for spectroscopic evaluation of gravity. Likewise, the damping wings of strong lines and other luminosity-sensitive line ratios are ruled out for this purpose.

A limited number of relatively uncontaminated atomic lines was selected as temperature critera for DY Per by KB97. We examined blending of these lines with molecular features in the spectrum of DY Per using the synthetic spectra of $C_{2}$ and CN molecules calculated for warm carbon star (see Sect. 3.2.2). We concluded that almost all lines provided in Table 2 of KB97 are the blends with lines of carbon bearing molecules. For example, the contribution of $\mathrm{CN}$ molecule in the Ni I line at $5235 \AA$ and the Al I line at $7835 \AA$ seems to be significant. The K I line at $7698 \AA$ is less affected by molecules (Fig. 6). However, only the Ti I line at $5238.6 \AA$ in this sample of lines is unblended in

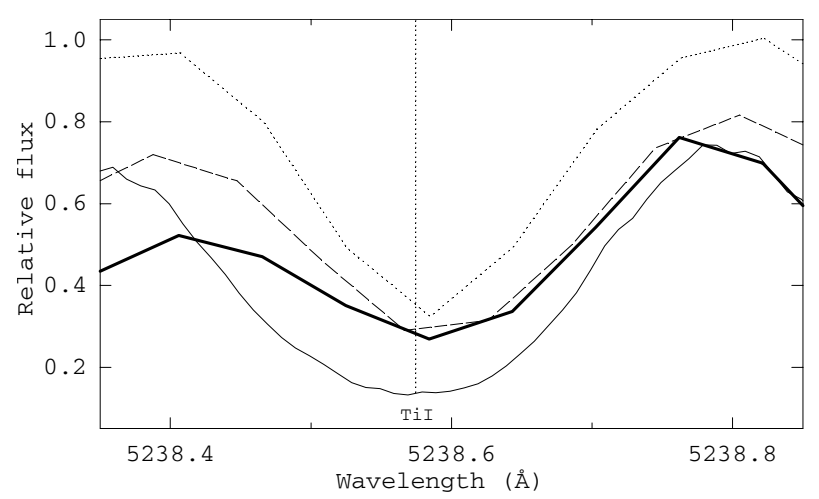

Fig. 7. Comparison of the Ti I line at $5238.57 \AA$ strengths in DY Per (thick solid line) and carbon stars of different effective temperature, $\mathrm{BD}+57^{\circ} 2161$ (dotted line), UV Cam (dashed line), and W CMa (thin solid line).

the spectrum of DY Per and comparison stars. Figure 7 compares the strength of this line in DY Per and three comparison stars. A correlation between the relative intensity and the effective temperature of star is clearly visible. The strength of this line is similar in two analyzed stars, DY Per and UV Cam, therefore, the effective temperatures should be similar. The spectrum synthesis of wavelengths region around 5238.6 $\AA$ for three different atmospheric models (temperatures) supports a good sensitivity of this line to the effective temperature of star (Fig. 8). The atmospheric parameters of three adopted models are close to those found for comparison stars $\mathrm{BD}+57^{\circ} 2161$, UV Cam, and W CMa. The solar titanium abundance was accepted in all models. We concluded that the effective temperature of DY Per is much higher than those for typical N-type carbon stars. This conclusion is supported by other line-strength studies. The line strength data for some selected atomic lines are summarized in Table 6. According to our analysis the temperature of $T_{\text {eff }} \sim$ $3500 \mathrm{~K}$ seems to be a good approximation for DY Per, in agreement with that estimated by KB97.

The requirement that neutral and ionized lines of an element yield the same abundance is commonly used to determine the surface gravity of a late-type star. We adopted a variant of this method. Unfortunately, due to the weakness and crowded spectrum the number of unblended lines of ions is extremely low. The pair of Ti lines near $4805 \AA$ (Fig. 9) is resolved and relatively uncontaminated in the spectra of all comparison stars. It should be a good criteria of gravity (luminosity). Unfortunately, this pair of lines was not resolved in the spectrum of DY Per. The reason seems to be a somewhat larger width (due to turbulence and/or rotation) of absorption lines in comparison with those for other carbon stars, however, neglected blends are not excluded. The sensitivity of s-process lines to gravity (luminosity) was tested too. There is a correlation between the ratio of light s-process lines Y II 5200.42 and Zr I 4805.87 (Table 6) and the luminosity 


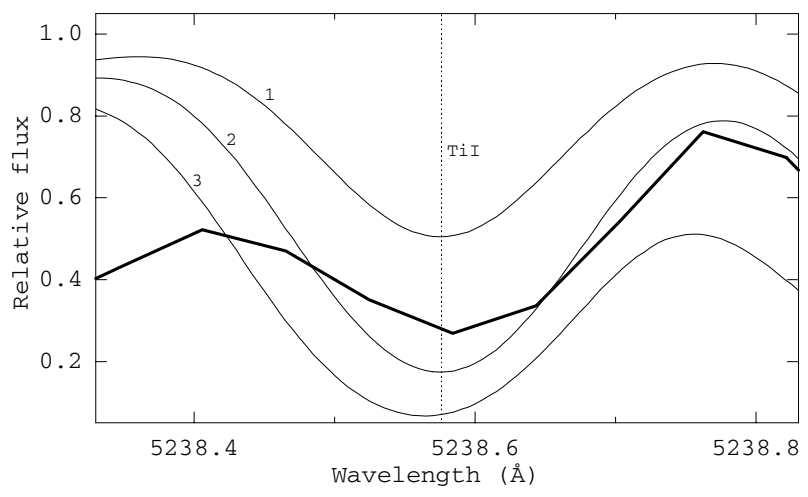

Fig. 8. High-resolution spectrum of DY Per (thick solid line) around the Ti I line at $5238.57 \AA$. The synthetic spectra for three atmospheric models with different effective temperature are provided: $4555 \mathrm{~K}$ (1), $3750 \mathrm{~K}(2)$, and $2750 \mathrm{~K}(3)$.

of star (Table 5) in the sample of analyzed carbon stars. This ratio is similar for two stars, DY Per and BD $+57^{\circ} 2161$. Thus for DY Per our analysis support the relatively low luminosity. According to Table 5 more luminous stars $\left(M_{\text {bol }} \sim-5\right)$ indicate larger Y II to Zr I ratio, in agreement with the crude simulation using atmospheric models.

Direct estimations of luminosities for the Galactic RCBs are absent. The best estimates are for the warm RCBs identified in the LMC: $M_{V}=-4$ to -5 mag. Alcock et al. (2001) suggested a relationship between absolute magnitude and effective temperature: the intrinsically brightest RCBs are the warm $(\sim 7000 \mathrm{~K})$ stars and the faintest are the cool $(\sim 5000 \mathrm{~K})$ stars. Thus DY Perlike stars in the LMC are the faintest with the maximum absolute brightness of $M_{V} \sim-2.5 \mathrm{mag}$. A crude estimation of the maximum luminosity for DY Per using the relationship between $(V-R)$ and $M_{V}$ found for RCBs in LMC (see Fig. 10 in Alcock et al. 2001) led to the maximum absolute brightness of $M_{V} \sim-2$ mag. This result is consistent with the $M_{V}$ estimates based upon pairs of neutral and ionized lines. The mean value of $(V-R) \sim 1.5$ at maximum light observed for DY Per (Alksnis $1994)$ and the extinction of $E(V-R)=0.32$ was adopted. $M_{V} \sim$ $-2 \mathrm{mag}$ and $A_{V}=1.5 \mathrm{mag}$ (Neckel \& Klare 1980) led to the distance of $1.6 \mathrm{kpc}$ for DY Per, however, a circumstellar reddening around DY Per was ignored.

\subsubsection{Hydrogen abundance and metallicity}

Strong Balmer absorption is an indicator of relatively high temperature of the carbon star: none of the typical N-type carbon stars have $\mathrm{H}_{\alpha}$ in absorption. However, in the spectra of typical R-type carbon stars the Balmer absorption is quite strong. The regions around lines of $\mathrm{H}_{\alpha}$ and $\mathrm{H}_{\beta}$ are crowded in the spectra of cool carbon stars and the identification of weak features due to Balmer lines was plagued. An inspection of another line of the Balmer series was not possible due to the low $S / N$ ratio in the blue spectral region. Careful analysis of the spectral region around $\mathrm{H}_{\alpha}$ for selected comparison stars and a confrontation with the synthetic spectra of $C_{2}$ and $\mathrm{CN}$ molecules shows that the intensity of $\mathrm{H}_{\alpha}$ for DY Per is much lower than that for carbon stars of similar temperature, for example, UV Cam (see Fig. 10). $\mathrm{H}_{\beta}$ is also much fainter than it should be for a star with a temperature of $T_{\text {eff }} \sim 3500 \mathrm{~K}$. Thus a significant hydrogen deficiency in the atmosphere of DY Per was verified.

In the spectrum of DY Per the lines of iron-peak elements are in general of similar intensity or slightly deficient relative to those for comparison stars (Fig. 11), therefore, the metallicity of DY Per is about solar.

\subsubsection{Carbon abundance and ${ }^{12} \mathrm{C} /{ }^{13} \mathrm{C}$ ratio}

An inspection of the high-resolution spectrum of DY Per shows that $C_{2}$ and $\mathrm{CN}$ lines are very strong relative to the comparison carbon stars. Extremely strong lines of $C_{2}$ Phillips system are visible, for example, in the near infrared region (Fig. 12). The shape of the spectrum of carbon star determine mainly the absolute abundances of $\mathrm{CNO}$ elements and the $\mathrm{C} / \mathrm{O}$ ratio. Thus enhanced lines of carbon bearing molecules relative to those for the typical carbon stars of different temperatures support a significant carbon enhancement in the atmosphere of DY Per.

Relative intensities of spectral features due to isotopic molecules were inspected using the $C_{2}$ Swan system band heads near $4740 \AA$ and selected lines of CN Red system $(2,0)$ at $8015 \AA$. Unfortunately, the strong bandheads are not a correct criteria of carbon abundance because they are beset with saturation problems. For example, the head of the Swan $(1,0)$ band comprises about sixty rotational lines which rapidly bring about saturation of the head intensity for moderate abundances (Wyller 1966). Indeed Alcock et al. (2001) found "....mixed evidence for the abundance of isotopic carbon ${ }^{13} \mathrm{C} . .$. " using bands of ${ }^{12} \mathrm{C}^{13} \mathrm{C}$ and ${ }^{13} \mathrm{CN}$ in their low resolution spectra for DY Per and DY Per-like stars in the LMC. The presence of bandhead of ${ }^{13} \mathrm{C}={ }^{13} \mathrm{C}$ at $4752 \AA$ in the high resolution spectra of DY Per and U Hya was not confirmed with certainty (Fig. 13). The absorption features near the position of ${ }^{13} \mathrm{C}^{13} \mathrm{C}$ bendhead are of similar strength in both stars, however, they are shifted in wavelength. Thus the absorption feature at $4752.0 \AA$ seems to be mainly due to the atomic lines of Na I at $4751.82 \AA, \mathrm{Cr}$ I at $4752.1 \AA$ and $\mathrm{Ni}$ I at $4752.11 \AA$. On the other hand, the isotopic bandhead at $4752.2 \AA$ is very strong in the J-type carbon star UV Cam with ${ }^{12} \mathrm{C} /{ }^{13} \mathrm{C}=4$. Therefore, the low isotopic ratio was rejected clearly for DY Per.

Single rotational lines of ${ }^{13} \mathrm{CN}$ molecule are favourable criteria of ${ }^{12} \mathrm{C} /{ }^{13} \mathrm{C}$ ratio in cool stars. A number of ${ }^{13} \mathrm{CN}$ lines were inspected in the spectrum of DY Per using wavelengths given by Wyller (1966). Unfortunately, most of the ${ }^{13} \mathrm{CN}$ lines are blended with much stronger lines of ${ }^{12} \mathrm{CN}$ and only a few weak isotopic features were detected. Clearly the isotopic lines are not enhanced relative to the carbon star U Hya and R Lep (Fig. 14). Thus ${ }^{12} \mathrm{C} /{ }^{13} \mathrm{C}$ ratio in the atmosphere of DY Per should be larger than that for all comparison stars. Accurate calculations of ${ }^{12} \mathrm{C} /{ }^{13} \mathrm{C}$ ratio will only be possible using spectra of very high resolution $/ S / N$ and atmospheric models.

\subsection{4. s-process elements}

A limited number of clean lines of neutron-capture elements were examined in the high-resolution spectrum of DY Per relative to the comparison carbon stars. The zirconium and yttrium lines in the spectrum of DY Per are weak in comparison with those for another carbon stars (Figs. 9 and 11). In the Table 6 the estimates of $\mathrm{Zr} \mathrm{I} / \mathrm{Ti}$ I and $\mathrm{Y}$ II/Fe I ratio display a lack of sprocessing in the evolution of DY Per, in agreement with that found by Dominy (1985). Thus the s-process estimates support the status of extreme RCBs for DY Per rather than the status of extreme thermally-pulsating asymptotic giant branch (TP-AGB) star. It is doubtful that DY Per could be the TP-AGB star because intrinsic carbon stars usually display a significant s-process enhancement (Abia et al. 2002). On the other hand, the distribution 
Table 6. Relative intensities and ratios of selected atomic lines in the high-resolution spectra of DY Per and comparison stars.

\begin{tabular}{cccccc}
\hline \hline Star & \multirow{2}{*}{ TiI 5238.57 } & \multirow{2}{*}{ KI 7698.98 } & YII 5200.42 & ZrI 4805.87 & YII 5200.42 \\
& & & FeI5191.46 & TiI 4805.42 & ZrI 4805.87 \\
\hline DY Per & 0.73 & 0.85 & 0.60 & 0.64 & 1.28 \\
UV Cam & 0.72 & 0.86 & 0.80 & 0.95 & 1.06 \\
U Hya & 0.80 & 0.90 & 0.93 & 1.40 & 1.05 \\
W CMa & 0.87 & - & 0.94 & 1.19 & 0.95 \\
BD +57²161 & 0.67 & 0.70 & 0.79 & 1.05 & 1.27 \\
\hline
\end{tabular}

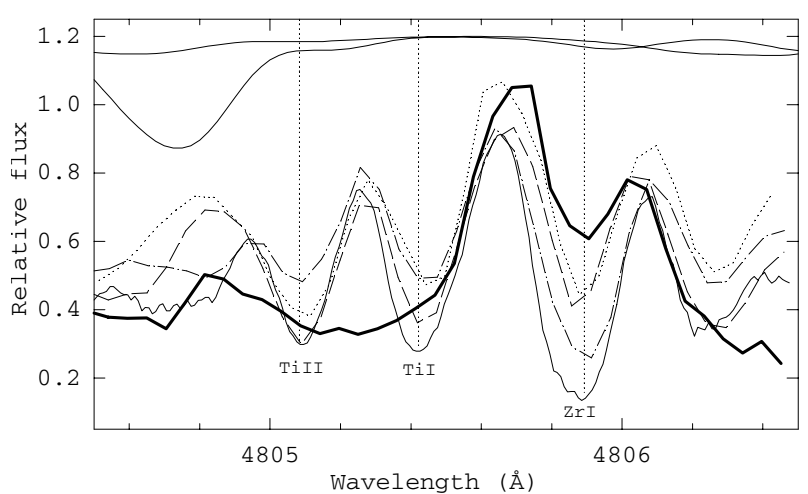

Fig. 9. High-resolution spectrum of DY Per (thick solid line) in the spectral region around three relatively clean lines of $\mathrm{Ti}$ and $\mathrm{Zr}$. The spectra of carbon stars with different effective temperature and luminosity, $\mathrm{BD}+57^{\circ} 2161$ (dotted line), U Hya (dashed-dotted line), UV Cam (dashed), and W CMa (thin solid line) are provided for comparison purposes.

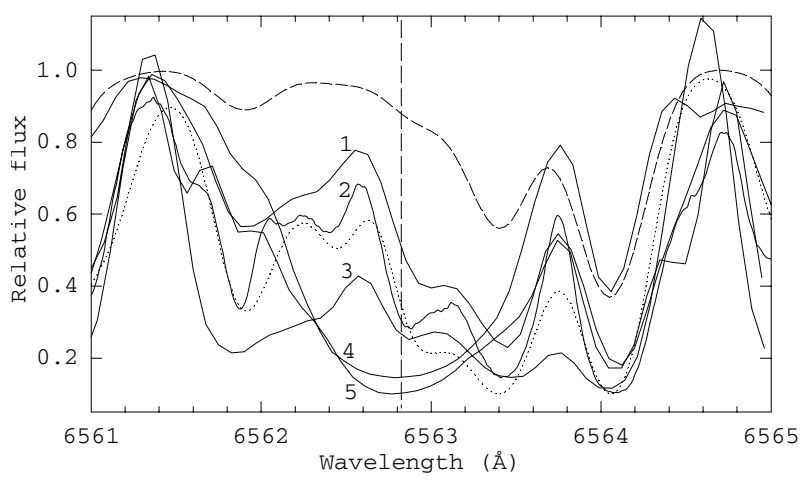

Fig. 10. High-resolution spectrum of DY Per (3) in the spectral region around $\mathrm{H}_{\alpha}$. The dashed vertical line indicate an accurate position of the Balmer line. The spectra of carbon stars of different effective temperatures and luminosities, U Hya (1), W CMa (2), UV Cam (5), and $\mathrm{BD}+57^{\circ} 2161$ (4), are provided for comparison purposes. The dashed and dotted lines represent synthesized spectra of a $\mathrm{CN}$ molecule for typical warm $(3700 \mathrm{~K})$ and cool $(2800 \mathrm{~K})$ carbon stars.

of the abundances of s-process elements [s/M] with respect to the metallicity parameter $[\mathrm{M}]$ does not show enhanced $[\mathrm{s} / \mathrm{M}]$ for analyzed RCBs of solar metallicity (Rao 2005).

\section{Evolutionary status}

RCBs as a class of stars are defined by two main characteristics. Firstly, the spectrum shows significant hydrogen deficiency and carbon enhancement. Secondly, typical light variations with irregular deep declines are present. DY Per fulfills both requirements therefore formally the status of RCBs is verified. However, it is known that all carbon stars (except R-type stars) lose mass (Olofsson et al. 1993). Moreover, deep

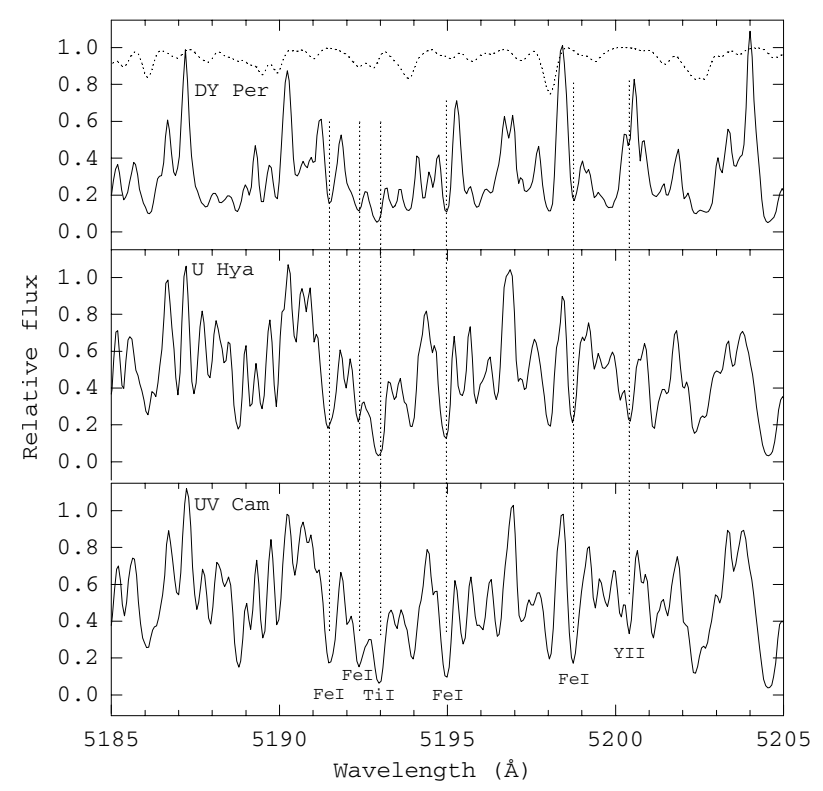

Fig. 11. High-resolution spectrum of DY Per (upper panel; solid line) in the spectral region from 5185 to $5205 \AA$ relative to the comparison stars. Dotted line represents the synthetic spectrum of ${ }^{12} \mathrm{CN}$ molecule for typical warm $(3700 \mathrm{~K})$ carbon star. The relatively uncontaminated lines of iron group elements and yttrium are marked with the dashed lines.

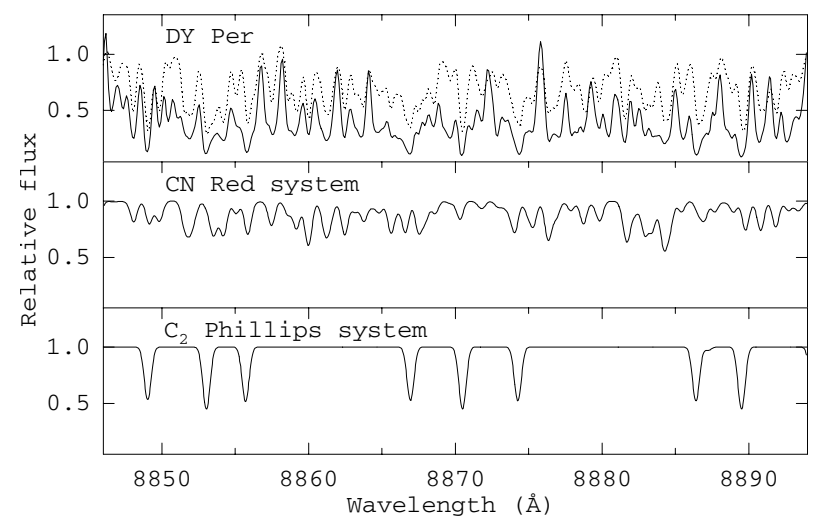

Fig. 12. High-resolution spectrum of DY Per (upper panel) in the spectral region from 8850 to $8890 \AA$. The dotted line represents the spectrum of cool carbon star U Hya. The synthetic spectra of the CN Red system and the $C_{2}$ Phillips system calculated for typical warm $(3700 \mathrm{~K})$ carbon star are given for comparison purposes.

fadings caused by dust formation are observed around these star. The fading and reddening of carbon Miras in the visible and the near infrared was observed by Lloyd Evans (2000 and references therein). The $J H K L$ photometry and spectroscopy of Mira R Lep and SRa variable V Hya revealed deep fadings which must result from absorption by newly formed circumstellar dust. The 


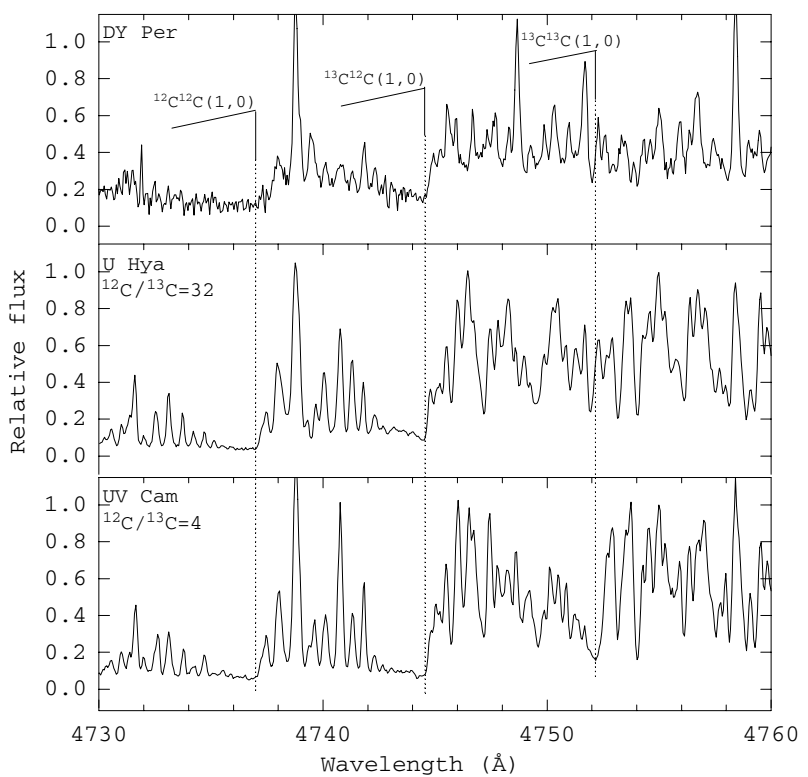

Fig. 13. High-resolution spectrum of DY Per (upper panel) in the spectral region around $C_{2}(1,0)$ Swan system bandheads. The spectrum of typical cool carbon star U Hya and J-type carbon star UV Cam are provided for comparison purposes.

emissions and/or P Cygni profiles appeared in $\mathrm{H}_{\alpha}$, Na I D1\&D2, $\mathrm{K} \mathrm{I}$, and $C_{2}$ during fading episodes in these stars (Lloyd Evans 2000). Thus some photometric and spectroscopic properties are similar for RCBs and cool pulsating carbon stars.

The evolutionary status of typical (warm) RCBs is not yet clear. Two major evolutionary paths leading to the RCBs, the double degenerate and the final helium flash, imply that these objects are evolved stars. In the final flash model, there is a close relationship between RCBs and planetary nebulae (PNe). The Galactic distribution and kinematic properties of RCB stars are important for identifying their progenitors. The metallicity of DY Per is near or slightly below the solar and the heliocentric radial velocity was found to be $-43.7 \mathrm{~km} \mathrm{~s}^{-1}$. Thus DY Per is clearly a disc object. The low scale height for the distance of $1.6 \mathrm{kpc}, z \sim 100 \mathrm{pc}$, support this conclusion. For the warm Galactic RCBs the use of magnitude at maximum brightness and the absolute magnitude of $M_{V}=-5$ mag results in $z=100$ $400 \mathrm{pc}$ (Iben et al. 1996). The true spatial distribution of RCB stars is uncertain, although the projected maps indicate a possible thick disk population (Zaniewski et al. 2005). The studies of planetary nebulae led to the average distance from the Galactic plane, $z \sim 400$ pc (Maciel \& Dutra 1992). However, the average $z$ for different groups was found to be a function of metallicity, varying from about $60 \mathrm{pc}$ at high metallicity to several kpc at low metallicity. Thus the Galactic distribution and kinematics for DY Per and warm RCBs are similar and are not in conflict with the final helium flash model.

\section{Conclusions and discussion}

A comprehensive analysis of the photometric and spectroscopic data gathered for DY Per near light maximums and at deep light decline during recent years strengthened the assumption that this carbon star could be the first extremely cool RCB star detected in our Galaxy. The blueshifted components at -197.3 and $-143.0 \mathrm{~km} \mathrm{~s}^{-1}$ in the profiles of sodium D lines, the veiling of strong absorption features and emissions in the Na I D1\&D2, $C_{2}$ $(1,0)$ bandhead and Ca I line at $4227 \AA$ detected at deep light

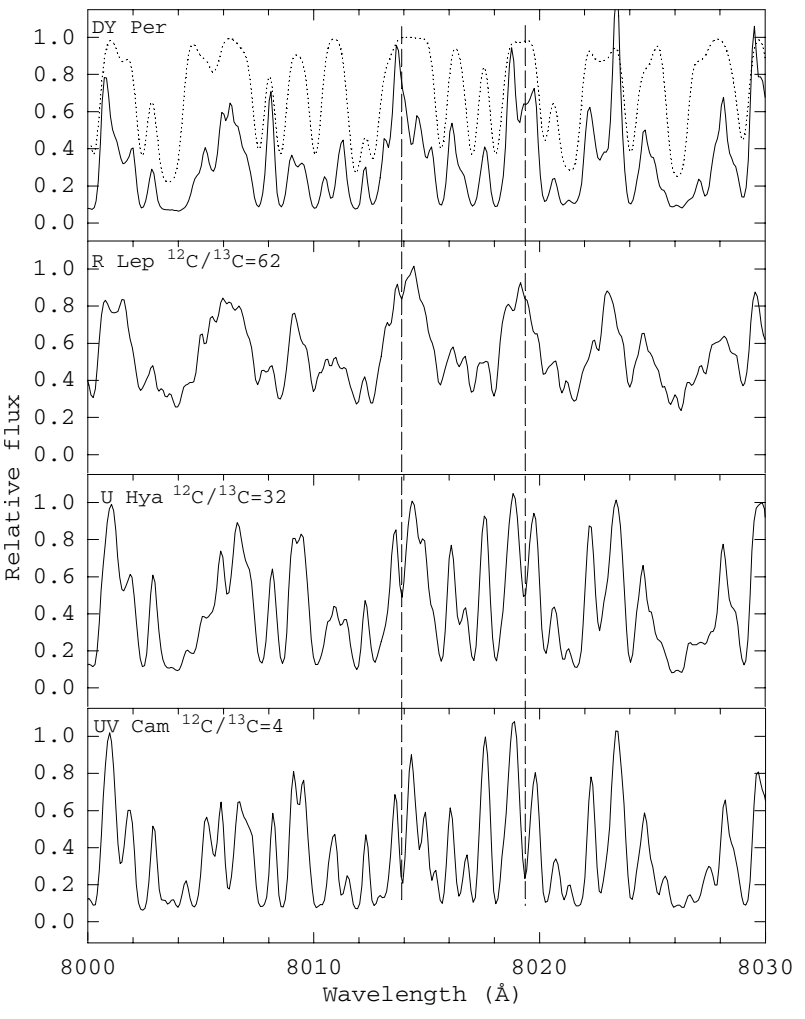

Fig. 14. High-resolution spectrum of DY Per (upper panel; solid line) relative to the comparison stars in the spectral region from 8000 to 8030 Å. Dotted line represents the synthetic spectrum of ${ }^{12} \mathrm{CN}$ molecule calculated for warm $(3700 \mathrm{~K})$ carbon star. The lines of ${ }^{13} \mathrm{CN}(2,0)$ marked with the dashed lines are not enhanced in the spectrum of DY Per.

decline support the existence of clouds of gas and dust ejected by DY Per. The blueshifted components display significant changes both in the radial velocity $\left(\sim 15 \mathrm{~km} \mathrm{~s}^{-1}\right)$ and shape between two seasons of observations in 1990 and 2002 (Začs et al. 2005a). A qualitative high-resolution spectroscopy of DY Per relative to the sample of carbon stars of different types support clearly the significant hydrogen deficiency, high carbon enhancement, and relatively high ${ }^{12} \mathrm{C} /{ }^{13} \mathrm{C}$ ratio. The s-process elements are not significantly enhanced, in agreement with what is observed for RCBs of about solar metallicity. However, the veiling of absorption lines at deep decline could be explained also because of hotter nearby star detected about 2'. 5 from DY Per.

The temperature of DY Per is extremely low in comparison with other Galactic RCB stars. The fine analysis of absorption lines with the goal to improve the effective temperature and gravity (luminosity) estimated for DY Per by Keenan \& Barnbaum (1997) support the low temperature, $T_{\text {eff }} \sim 3500 \mathrm{~K}$, and a bright giant luminosity, $M_{V} \sim-2 \mathrm{mag}$, for this unique star. DY Per demonstrate long period semiregular large amplitude pulsations typical for cool carbon-rich AGB stars with a period of 792 days (Alksnis et al. 2002). The simulations of pulsations using models of RCBs provided by Weiss (1987) provided the period of about 800 days for the extremely cool model with $T_{\text {eff }}$ of about $3600 \mathrm{~K}$. This supports the conclusion that DY Per could be an extremely cool RCB.

The temperature and luminosity estimated for DY Per are similar to the parameters estimated for the coolest RCBs in LMC. However, Alcock et al. (2001) found evidence for a significant amount of ${ }^{13} \mathrm{C}$ in their spectra. Moreover, the DY Perlike stars of LMC display only small amplitude photometric 
pulsations. Although the two major scenarios of RCB formation do not require a similar temperature and light behaviour for all RCBs, the path of evolution of these subgroups could be different. High-resolution spectroscopy is needed for DY Per-like stars of LMC, SMC and Galactic bulge to specify its chemical characteristics and clarify the relationship with DY Per.

The Galactic distribution, radial velocity and chemical composition indicate clearly that DY Per is a disk object, in agreement with that observed for part of warm Galactic RCBs and PNs. We conclude that it is unlikely that DY Per is an extreme TP-AGB carbon star due to the absence of significant s-process enhancement and the hydrogen deficiency.

The anonymous star detected at deep light decline about 2 '.5 from DY Per does not vary appreciably in the $B$ and $V$ bands, $B=17.8$ mag. With observed colour indices $(B-V)=1.00$ and $(V-R) \simeq 0.6$ it may be a foreground G0 dwarf, however, the physical connection between these stars is not excluded. The distance to both stars seems to be similar, $d \simeq 1.5 \mathrm{kpc}$.

Acknowledgements. We are grateful to Y. T. Chen, P. S. Chiang, H. C. Lin, Z. Y. Lin, and M. C. Tsai for their help with the photometric observations. The referee, G. C. Clayton, is thanked for his critical comments on the paper. The Mutual Fund of scientific collaboration among Taiwan (ROC), Latvia and Lithuania is thanked for support. We acknowledge with thanks the variable star observations from the AAVSO International database contributed by observers worldwide and used in this research.

\section{References}

Abia, C., Dominguez, I., Gallino, R., et al. 2002, ApJ, 579, 817 Alcock, C., Allsman, R. A., Alves, D. R., et al. 2001, ApJ, 554, 298
Alksnis, A. 1994, Baltic Astron., 3, 410

Alksnis, A. 2003, Baltic Astron., 12, 595

Alksnis, A., Larionov, V. M., Larionova, L. V., \& Shenavrin, V. I. 2002, Baltic Astron., 11, 487

Bergeat, J., Knapik, A., \& Rutily, B. 2002, A\&A, 390, 967

Clayton, G. C. 1996, PASP, 108, 225

Dominy, J. F. 1985, PASP, 97, 1104

Iben, I., Tutukov, A. V., \& Yungelson, L. R. 1996, ApJ, 456, 750

Johnson, H. L. 1966, ARA\&A, 4, 193

Keenan, P. C., Barnbaum, C. 1997, PASP, 109, 969 (KB97)

Kerschbaum, F., \& Hron, J. 1992, A\&A, 263, 97

Kharchenko, N. V., Piskunov, A. E., Röser, S., Schilbach, E., \& Scholz, R. D. 2005, A\&A, 438, 1163

Kramer, K. E., Sloan, G. C., Wood, P. R., Price, S. D., \& Egan, M. P. 2005, ApJ, 631, L147

Lambert, D. L., Gustafsson, B., Eriksson, K., \& Hinkle, K. H. 1986, ApJS, 62, 373

Lawson, W. A., Cottrell, P. L., Kilmartin, P. M., \& Gilmore, A. C. 1990, MNRAS, 247, 91

Lloyd Evans, T., 2000, in The carbon star phenomenon, ed. R. F. Wing (Dordrecht: Kluwer), IAU Symp., 177, 367

Maciel, W. J., \& Dutra, C. M. 1992, A\&A, 262, 271

Metlov, V. G. 2004, Astron. Astrophys. Trans., 23, 197

Neckel, Th., \& Klare, G. 1980, A\&AS, 42, 251

Olofsson, H., Eriksson, K., Gustafsson, B., \& Carltrom, U. 1993, ApJS, 87, 267

Rao, N. K. 2005, in Cosmic Abundances as Records of Stellar Evolution and Nucleosynthesis in honor of David L. Lambert, ed. T. G. Barnes, \& F. N. Bash, ASP Conf. Ser., 336, 185

Rao, N. K., \& Lambert, D. L. 1993, AJ, 105, 1915

Wallerstein, G., \& Knapp, G. R. 1998, AR\&A, 36, 369

Weiss, A. 1987, A\&A, 185, 178

Wyller, A. A. 1966, ApJ, 143, 828

Začs, L., Chen, W. P., Alksnis, O., et al. 2005a, A\&A, 438, L13

Začs, L., Schmidt, M., Musaev, F., Galazutdinov, G. A., \& Sperauskas, J. 2005b, A\&A, 441, 303

Zaniewski A., Clayton, G. C., Welch, D. L., et al. 2005, AJ, 130, 2293 